Ophthalmologica 1943;105:I-IV

\title{
Contents, Vol. 105, 1943
}

\section{Index}

Amsler, M., et Verrey, F., De Гutilité pratique de la ponc-

tion de la chambre antérieure 144

Arruga, H., Le traitement de Thémorragie expulsive par la

neurotomie optico-ciliaire 106

Bruckner, R., Auge und Cholinesterase

Bürki, E., Zu $\gamma$ Kenntnis des Retothelsarkoms der Orbita

Bürkí, E., und Schmíd, A. E., Histochemis < che Untersuchun-gen zum Nachweis und zur

Lokalisation des Vitamin Gim Auge 65, 121

Delbos, R., vide Redslob, E.

Delogé, La reeducation visuelle 91

Girardet, M., Une nouvelle famille de cataracte poussié-reuse centrale (cataracta centralis

pulverulenta)(Planche I) 24

Goldmann, H., Objektive Sehschärfenbestimmung . . 240

Halbertsma, K. T. A., Papilloma corneae 299

Lieux, et Saint-Martin, R. de, Nouveau cas de blessure du

trijumeau par éclat de grenade rétroorbitaire. .

Poleff, L., Der wirkliche Wert der Sulfamidotherapie bei

Trachom 156

Rapin, M., Restauration des paupières inférieure et supé-

rieure 233

Redslob, E., et Delbos, R., Ophtalmie sympathique sans

ophtalmie sympathisante apparente 83

Saint-Martin, R. de, Sur un nouveau cas d'hémianopsie bi-

temporale traumatique

289 vide Lieux.

Schmid, A. E., vide Bürkí, E.

Verrey, F., Un dispositif pour la centrifugation capillaire $151 \quad$ vide Amsler, M.

Wagenaar, J. W., Ueber eine einfache Methode, den Augen-hintergГund ohne stereoskopische

Einrichtung stereo-skopisch zu sehen 13

Weekers, R.\} Nouvelle contribution à $\Gamma$ étude du glaucomeincomplet. L'excavation glaucomateuse monosympto-matique 307

UEBERSICHTSREFERATE - REVIEWS - REVUES

Babel, J., Uvée. (Années 1939 à 1941) .... 268, 318

Coppez, L., Nystagmus. 1939 . 107

Süllmann, H., Biochemie des Auges (Berichtszeit 1938 bis

1942) 175

GESELLSCHAFTSBERICHTE - SOCIETY TRANSACTIONS - SOCIÉTÉS

Sitzungsbericht der Niederländischen Ophthalmologischen Gesellschaft 
in Utrecht am 13. u. 14. Juni 1942. Von C. 0. Roelofs, Amsterdam 49

Schweizerische Ophthalmologische Gesellschaft. 35. Jahresversammlungam 12. bis 13.

September 1942 in Chexbres. - Société Suissed'Ophtalmologie. 35e Assemblée générale les 12/13 septembre 1942à Chexbres. E.B.Streiff, Geneve 219

Vereinigung der Basler Augenärzte. Sitzung am 10. Dezember 1942 in der Universitäts-Augenklinik in Basel ...... 283

B. BUCHBESPRECHUNGEN - BOOKS REVIEW - LIVRES NOUVEAUX 119

231 